CERN-EP/98-09

January 9 th, 1998

\title{
First Observation of Cherenkov Ring Images using Hybrid Photon Detectors
}

\author{
E. Albrecht ${ }^{2)}$, G. Barber ${ }^{4)}$, J.H. Bibby ${ }^{5)}$, N.H. Brook ${ }^{3)}$, A. Duane ${ }^{4)}$, M. French ${ }^{7)}$, V. Gibson ${ }^{1)}$, \\ R. Giles ${ }^{5)}$, A.W. Halley ${ }^{3)}$, N. Harnew ${ }^{5)}$, M. John ${ }^{4)}$, D. G. Miller ${ }^{4}$, V.O'Shea ${ }^{3)}$, R. Schomaker ${ }^{6)}$, \\ N. Smale ${ }^{5)}$, D. Websdale ${ }^{4)}$, G. Wilkinson ${ }^{2)}$, S.A. Wotton ${ }^{1)}$.
}

\begin{abstract}
A Ring-Imaging Cherenkov detector, equipped with Hybrid Photon Detectors, has been operated in a charged-particle beam. Focussed ring images from various particle types were detected using silica aerogel, air and $\mathrm{C}_{4} \mathrm{~F}_{10}$ gas radiators. The detector, a prototype for the CERN LHC-B experiment, is described and first observations are reported.
\end{abstract}

\footnotetext{
1) University of Cambridge, Cavendish Laboratory, Madingley Road, Cambridge CB3 OHE, UK.

2) CERN, PPE Division, 1211 Geneva 23, Switzerland.

3) University of Glasgow, Department of Physics, Glasgow G12 8QQ, UK.

4) Imperial College of Science Technology \& Medicine, Blackett Laboratory, Prince Consort Road, London SW7 $2 A Z$, UK.

5) University of Oxford, Department of Nuclear Physics, Keble Road, Oxford OX1 3RH, UK.

6) Delft Electronic Products BV, 9300AB Roden, NL.

7) Rutherford Appleton Laboratory, Chilton, Didcot, Oxon OX11 OQX, UK.
} 


\section{Introduction}

This paper reports on first results from a Ring Imaging Cherenkov (RICH) counter which uses an array of Hybrid Photon Detectors (HPD's) to detect the Cherenkov photons. The RICH is a prototype of the upstream detector for the LHC-B experiment [1], proposed for the CERN Large Hadron Collider. The LHC-B detector is a single-arm spectrometer covering polar angles between approximately 10 and $350 \mathrm{mrad}$. The forward geometry provides high acceptance for $B$ hadrons, which are produced predominantly in the forward (and backward) directions.

The aim of the LHC-B experiment is to make precise measurements of $\mathrm{CP}$ violation in $B$ decay. The particle identification provided by two RICH detectors is crucial, firstly by reducing backgrounds in selected final states, and hence minimizing systematic errors on the CP measurements, and secondly by providing an efficient flavour tag (using kaons) of $B^{0}$ or $\overline{B^{0}}$. The RICH detectors, shown in the experimental layout of Fig. 1 , provide $\pi / \mathrm{K}$ separation over the momentum range $1-150 \mathrm{GeV} / c$.

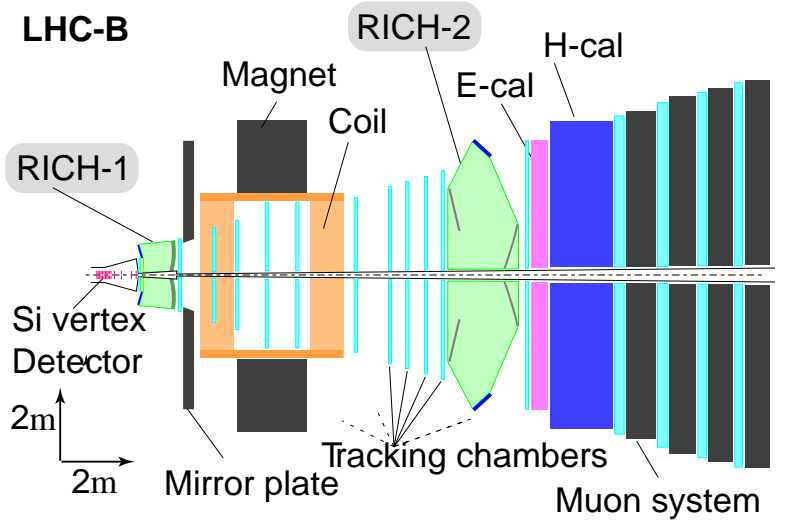

Figure 1: The LHC-B spectrometer.

A schematic diagram of the upstream RICH detector [2] (RICH-1) is shown in Fig. 2. It combines gas and aerogel radiators and is situated in front of a $3 \mathrm{Tm}$ dipole magnet. The aerogel radiator is placed against the entrance window of a second, $\mathrm{C}_{4} \mathrm{~F}_{10}$, gas radiator. A spherical mirror of focal length $1000 \mathrm{~mm}$, tilted by $\sim 250 \mathrm{mrad}$, reflects the Cherenkov photons out of the spectrometer acceptance onto an array of photodetectors. The refractive indices of the aerogel $(n=1.03)$ and $\mathrm{C}_{4} \mathrm{~F}_{10}$ $(n=1.0014)$ allow $\pi / \mathrm{K}$ separation over the momentum range $1-65 \mathrm{GeV} / c$. The expected mean numbers of detected photoelectrons are $\sim 15$ and $\sim 55$, respectively, for a saturated $(\beta=1)$ track.

Ypsilantis and Seguinot [3] proposed that the LHC-B RICH counters use an array of HPD's to detect the Cherenkov light. These devices, developed by De Salvo [4], in collaboration with DEP ${ }^{1)}$,

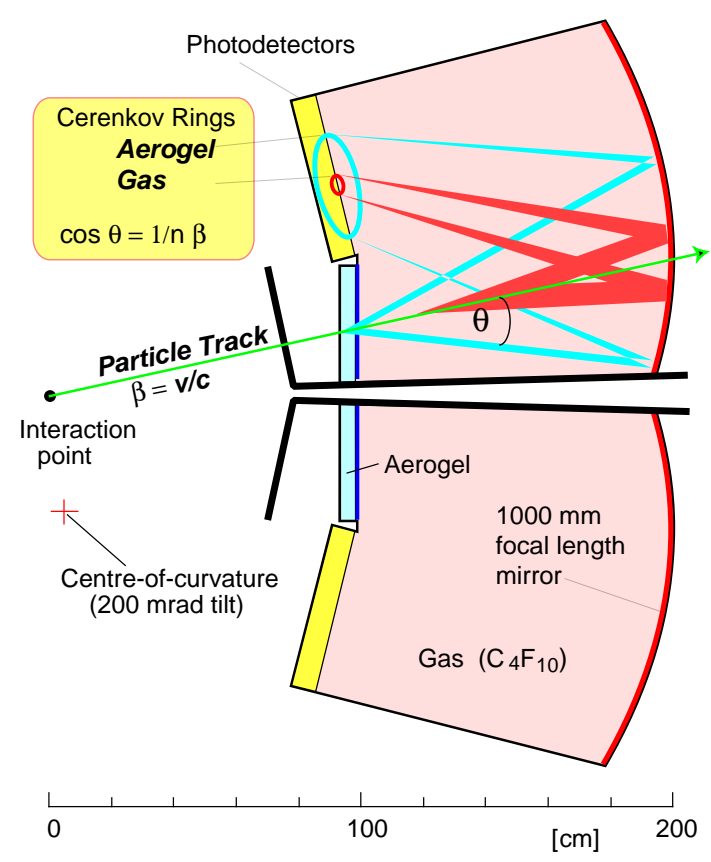

Figure 2: Layout of the LHC-B RICH-1 detector, (the LHC beams pass horizontally through the vacuum-pipe at the centre).

involve the acceleration of photoelectrons through several kilovolts from a photocathode onto a silicon detector. HPD's are sensitive to visible and nearUV light and the silicon pixels give unambiguous 2-dimensional space points. They have many advantages when used to detect photons in $\mathrm{RICH}$ detectors at the LHC :

- They have low noise, high quantum efficiency and good single-photon sensitivity; however the signal charge resulting from one photoelectron is relatively small, $\sim 3000$ electrons.

- They can provide large-area coverage, whilst the silicon segmentation gives the necessary detector granularity. A pixel size of $2 \times 2 \mathrm{~mm}^{2}$ is proposed for the LHC-B RICH detectors.

- The intrinsic signal is fast enough to be used at the $25 \mathrm{~ns}$ LHC bunch-crossing frequency. It is proposed that the two LHC-B RICH detectors will use a total of 250 large-area HPD's, each with an $11 \mathrm{~cm}$ diameter photocathode, leading to 500,000 silicon-pixel channels in total. Hexagonal close-packing of the HPD's will provide $80 \%$ active area over the detector surface. The HPD devices used in the prototype tests reported here were manufactured by DEP. They have an hexagonal array of 61 pixels, approximately $2 \times 2 \mathrm{~mm}^{2}$, with a relatively small active photocathode area $(18 \mathrm{~mm}$ diameter). Developments for producing an HPD with

\footnotetext{
1) Delft Electronics Products BV, 9300AB Roden, NL.
} 
a large-area photocathode are underway [5].

The radiators of RICH- 1 have been chosen to match the $\pi / \mathrm{K}$ momentum range of interest. The use of high clarity samples of aerogel as radiator material in RICH detectors has been recently demonstrated $[6,7]$. The attraction of aerogel is that it can be produced with a refractive index in the range 1.01-1.10, suitable for the low end of the momentum range (down to $1 \mathrm{GeV} / c$ ) over which particle identification is required for LHC-B. Samples of aerogel with $n \approx 1.03$ have been used in the studies reported here. $\mathrm{C}_{4} \mathrm{~F}_{10}$ gas is chosen for its high refractive index and low dispersion; it is the heaviest fluorocarbon that remains gaseous at room temperature.

This paper describes the design, construction and first results of a prototype of the LHC-B RICH1 detector. Section 2 describes the prototype detector, its mechanical construction, the optics, the HPD's, the aerogel and gas radiators, the gas system, the electronics readout and data acquisition. Section 3 presents preliminary results from the prototype in a charged-particle beam. A summary is given in Section 4, including plans for future analysis and $\mathrm{R} \& \mathrm{D}$.

\section{The Prototype RICH detector \\ 2.1 Overview}

This section describes the design of the prototype RICH-1 detector, a milestone in the progression towards the preparation of the LHC-B Technical Proposal. The aims of the prototype experimental programme are as follows :

- To demonstrate the single-photon sensitivity of commercial 61-pixel HPD's and to measure their spatial precision and the signal/noise ratio.

- To operate the RICH-1 prototype in a charged-particle beam and to detect simultaneously the Cherenkov rings from the $\mathrm{C}_{4} \mathrm{~F}_{10}$ gas and the aerogel radiators.

- To measure the precision in the Cherenkov angle of detected photons using a full-scale prototype of the RICH-1 device.

- To study Rayleigh scattering in the aerogel radiator using UV filters.

- To measure the effects due to ionizing particles traversing the HPDs.

A planar array of seven 61-pixel HPD's is used to detect the Cherenkov rings produced in both aerogel and $\mathrm{C}_{4} \mathrm{~F}_{10}$ radiators. The rings are focussed by a $240 \mathrm{~mm}$ focal length mirror, giving a $\frac{1}{4}$-scale prototype of the RICH- 1 detector. This is referred to below as Configuration-1. The full-scale prototype (Configuration-2) uses a $1143 \mathrm{~mm}$ mirror to focus rings from $\mathrm{C}_{4} \mathrm{~F}_{10}$ onto an array of six 61-pixel HPD's.

\subsection{HPD detectors}

The Hybrid Photon Detector uses a visiblelight photocathode, in which the photoelectrons are accelerated to bombard a segmented silicon detector. At the time this protoype project was conceived only single-channel and 7-pixel HPD's were available commercially from DEP. This same company was contracted to manufacture a 61 -pixel $\mathrm{HPD}^{2)}$, and has supplied all the devices used in the tests reported here. A sketch of the HPD tube and its silicon-pad layout is shown in Fig. 3.

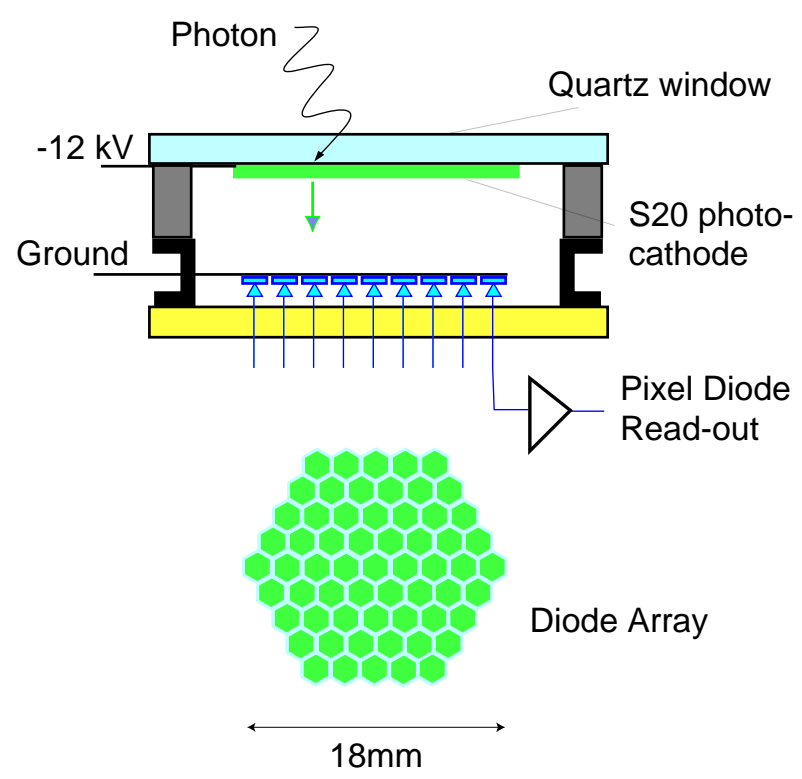

Figure 3: Sketch of 61-pixel HPD and the hexagonal array of silicon pads.

The photocathode is an S20 type (semitransparent $\mathrm{SbNa}_{2} \mathrm{KCs}$ ), deposited on the inside surface of a $2 \mathrm{~mm}$ thick fused-silica window. The silicon diode detector is processed on a $300 \mu \mathrm{m} \mathrm{n}$ type substrate and the p-type implant is segmented as an hexagonal array with pad dimension of $2 \mathrm{~mm}$ flat-to-flat. The photoelectrons travel $12 \mathrm{~mm}$ from the photocathode (at a potential of $-12 \mathrm{kV}$ ) to the back (ohmic) surface of the silicon. This surface implant has been thinned to $\sim 100 \mathrm{~nm}$ thickness. The diodes are reverse biased at $60 \mathrm{~V}$ (more than is required for depletion). Ohmic contacts to the diode pads are provided by a $2.54 \mathrm{~mm}$ pitch, 80 -pin grid array which feeds through into the vacuum tube via a ceramic carrier, solder bump bonded to the metallized p-type pads.

Apart from routine measurements performed at the DEP factory (HV test, diode leakage currents and photocathode response) no characterization nor selection of the HPD was made prior to their installation in the prototype RICH detector.

\footnotetext{
2) A product issuing from a collaborative development project with INFN (Italy) and Southampton University (UK), with funding from the European Union.
} 
A photograph of the HPD array during installation is shown in Fig. 4.

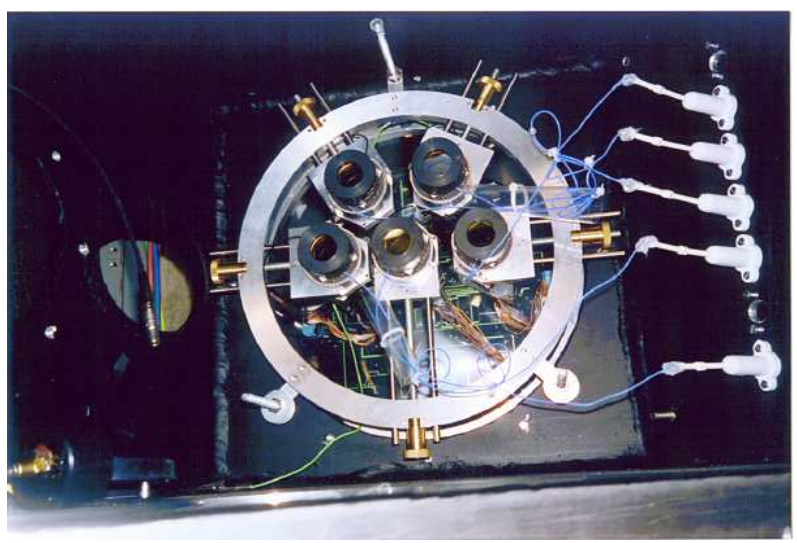

Figure 4: Photograph of the HPD array during assembly.

\subsection{Mechanics and Optics \\ 2.3.1 Configuration-1}

The prototype Cherenkov counter is designed as a vacuum vessel to simplify the gas-filling procedure. Configuration-1, shown schematically in Fig. 5, is a $\frac{1}{4}$-scale prototype of the LHC-B RICH-1 detector. It is constructed using aluminium alloy ${ }^{3}$ ) plate, folded and welded at the box edges. The box is black-anodized to reduce reflection of stray light. A $10 \mathrm{~mm}$ thick removable backplate supports the mirror. It is bolted to the box and sealed with a rubber O-ring. Portholes (not shown in the figure) are machined into the side and backplate. These fulfil different functions, e.g. a standard photo-multiplier tube can be mounted to check for light leaks. The ports are sealed using blank plates and O-rings when the box is connected to the pump or to the gas system.

The beam particles enter the box along a tube of $90 \mathrm{~mm}$ internal diameter, passing through a mylar window of $250 \mu \mathrm{m}$ thickness. The beam axis intersects the mirror centre at an angle of $18^{\circ}$ to the mirror axis. Three micrometer screws permit adjustment of the mirror angle about an axis in the plane, and perpendicular to the plane of the drawing (Fig. 5). A movement of $1 \mathrm{~mm}$ at the micrometer screw results in a shift of $5 \mathrm{~mm}$ of the image at the detector plane, which is fixed at a distance of $250 \mathrm{~mm}$ from the mirror centre. Initial alignment of the mirror was performed using a laser beam, incident along the beam axis and reflected by the mirror onto the detector plane.

The gas Cherenkov radiator is contained within a volume of length $400 \mathrm{~mm}$ between the mylar window and the mirror. This volume is filled either with air or $\mathrm{C}_{4} \mathrm{~F}_{10}$. Samples of aerogel radiator

\footnotetext{
3) BS-HS30
}

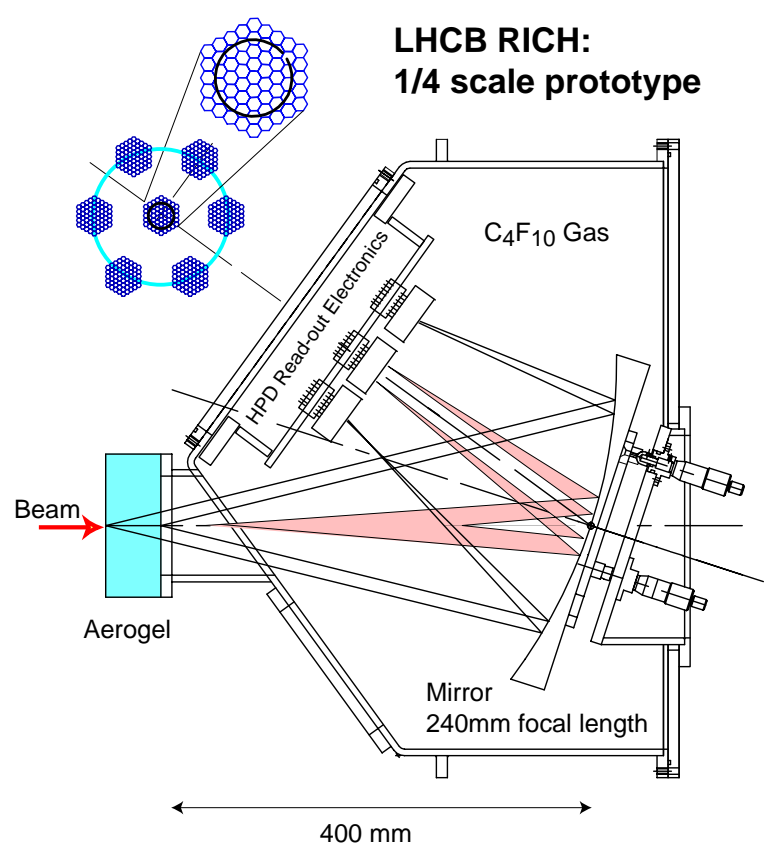

Figure 5: Configuration-1: the $\frac{1}{4}$-scale prototype RICH vessel.

are placed at the beam entrance window, upstream of the gas radiator. When used together with $\mathrm{C}_{4} \mathrm{~F}_{10}$, the mylar window is always downstream of the aerogel, and thus filters out the short $(<350 \mathrm{~nm})$ wavelength Cherenkov light. When used together with air, data were taken without and with filters (mylar and borosilicate glass were used).

The HPD's are arranged at the detector plane as shown in Fig. 5. The inner HPD is positioned to detect the image from the gas radiator, while the aerogel image is detected by the outer array. The HPD's are mounted on a plate, machined with holes at appropriate locations, through which the HPD sockets (see section 2.7) pass with an O-ring "piston" seal. Initial tests, performed using air at atmospheric pressure, allowed individual HPD's to move on adjustable supports, shown in Fig. 4. This permitted a measurement of the ring image positions from our aerogel samples (the precise value of the refractive index was not previously known). The holes in the mounting plate could then be machined accurately.

Spherical glass mirrors were fabricated commercially ${ }^{4)}$. The concave surface was machined from a solid piece of glass using a diamond cutter; the surface was then aluminized and coated with a thin magnesium flouride layer. The mirror used in Configuration- 1 has a focal length of $240 \mathrm{~mm}$ and a $310 \mathrm{~mm}$ diameter.

4) Optical Works Ltd. Newquay, Cornwall TR7 1HX,UK 


\subsubsection{Configuration-2}

Configuration-2, shown in Fig. 6, is designed as a full-scale prototype for the LHC-B RICH-1 detector. Extensions are added to Configuration1 to provide for a $1000 \mathrm{~mm}$ gas radiator length. The HPD's are mounted at the focal plane of a mirror having a $1143 \mathrm{~mm}$ focal length and diameter of $120 \mathrm{~mm}$. The reflectivity of the mirror was measured $^{5)}$ and found to be uniform in the visible spectrum, at approximately $85 \%$, falling to $\sim 40 \%$ at $160 \mathrm{~nm}$.

The HPD mounting plate was machined to allow Cherenkov rings from the $\mathrm{C}_{4} \mathrm{~F}_{10}$ radiator to be focussed on the photocathodes of the six outer HPD's. The aim of this setup is to evaluate the precision achievable in measurement of the Cherenkov angle, and hence of the particle identification performance of the LHC-B RICH-1 detector.

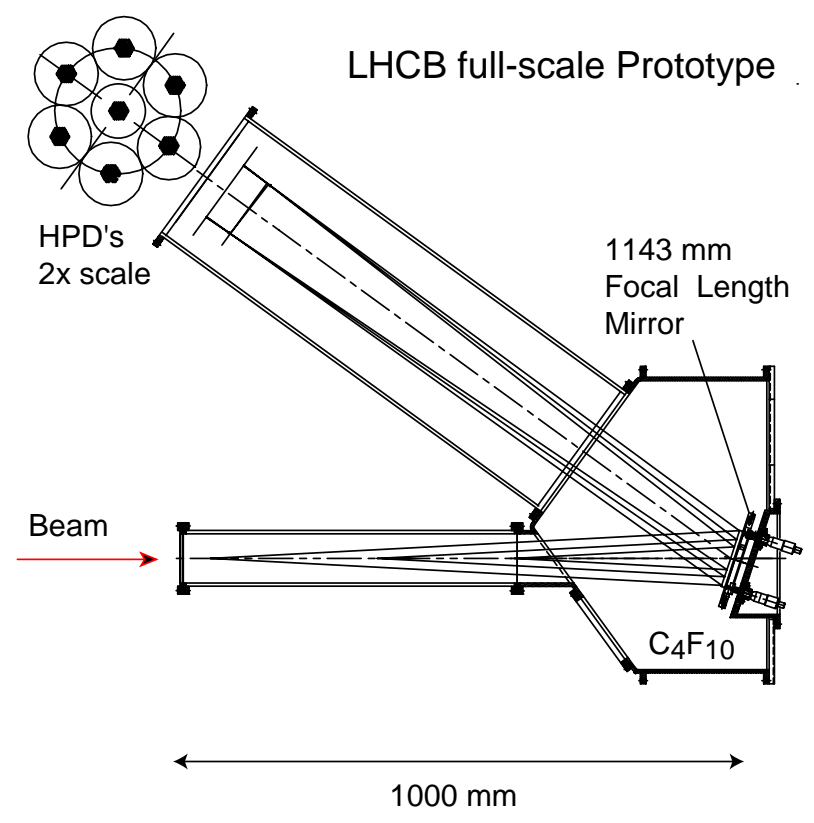

Figure 6: Configuration-2: prototype of LHC-B RICH-1 Detector.

\subsection{Aerogel samples}

Silica aerogel is proposed as a radiator in the LHCB RICH-1 for identification of low momentum $(<15 \mathrm{GeV} / c)$ particles. A refractive index $n \sim 1.03$ provides optimal velocity resolution. We were supplied with samples of hydrophobic aerogel from two sources:

Sample 1: $110 \times 110 \mathrm{~mm}^{2}$ surface $\times 18 \mathrm{~mm}$ thickness, produced at KEK ${ }^{6}$,

Sample 2: $110 \times 110 \mathrm{~mm}^{2}$ surface $\times 11 \mathrm{~mm}$ thickness, produced by Matsushita ${ }^{7)}$ for the HERMES experiment at DESY.

5) A.Braem, CERN

6) KEK Laboratory, Japan; supplied by J.Seguinot, Collège de France

7) Matsushita Company, Japan, supplied by E.Nappi, Univ. of Bari, Italy
These samples were used as Cherenkov radiators, positioned as in Fig. 5, $425 \mathrm{~mm}$ upstream of the mirror centre. Both samples were specified as having a nominal refractive index, $n \sim 1.03$. The transmission characteristics were measured and are shown in Fig. 7, as a function of the incident wavelength, $\lambda$. The curves plotted on this figure are simple parameterizations of the wavelength dependence of the transmission coefficient $T$, motivated by Rayleigh scattering:

$$
T \propto \exp \left(-c L \lambda^{-4}\right)
$$

where $L$ is the length of the aerogel sample and $c$ is a "clarity" coefficient for the sample. Good fits to the measurements are obtained with values of $c$ of order $0.008 \mu \mathrm{m}^{4} / \mathrm{cm}$.

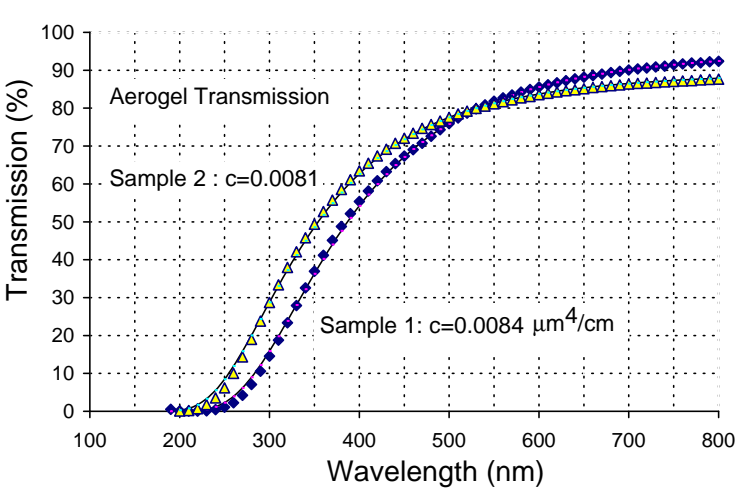

Figure 7: Transmission characteristics of aerogel samples.

\subsection{Gas system for Perfluorobutane $\left(\mathbf{C}_{4} \mathbf{F}_{10}\right)$}

A gas recirculation system was constructed to demonstrate the operation of the prototype $\mathrm{RICH}$ with the fluorocarbon gas $\mathrm{C}_{4} \mathrm{~F}_{10}$, the preferred radiator for RICH-1. The recirculation system is necessary to maintain high gas purity in the detector. The purity of the $\mathrm{C}_{4} \mathrm{~F}_{10}$ is an important consideration for our application, especially for operation in the ultraviolet (the HPD sensitivity covers the spectral range from $170-700 \mathrm{~nm}$ ). The ability of the gas system to recover the gas for re-storage is also a necessary feature, due to its high cost and for environmental considerations.

A schematic of the gas system is shown in Fig. 8. Its design is based on the principles used in the DELPHI forward RICH gas system [8]. The purification relies upon liquefaction of the $\mathrm{C}_{4} \mathrm{~F}_{10}$ gas and its subsequent storage. Residual contaminants such as nitrogen, argon and $\mathrm{CO}_{2}$ are left in the gaseous state, enabling these gases to be 
purged from the region above the $\mathrm{C}_{4} \mathrm{~F}_{10}$ liquid. Prior to liquefaction, water vapour is removed with a molecular-sieve drier. The liquefied $\mathrm{C}_{4} \mathrm{~F}_{10}$ is then re-heated to provide a supply of pure gas to the detector. During normal circulation, the pressure in the RICH is maintained within a range of $\pm 20 \mathrm{mbar}$, between 500 and 1300 mbar absolute.

A domestic freezer is used to liquefy the gas. The freezer is operated at a temperature $<-20^{\circ} \mathrm{C}$; this determines the vapour pressure of the gaseous $\mathrm{C}_{4} \mathrm{~F}_{10}$ above the liquid. The system is provided with sensors which monitor the gas pressure and flow, freezer and heater temperature, water and oxygen level, and solenoid valve operation. The gas system and RICH vessel are vacuum-pumpable down to $10 \mathrm{mbar}$, to allow an efficient purge of gas prior to filling.

\subsection{HPD High Voltage}

The high voltage for the HPD's is derived from a commercial low-ripple power supply unit ${ }^{8)}$ which is mounted on the outside of the prototype vessel. The output voltage can be varied between $0-15 \mathrm{kV}$ and is decoupled to ground via $500 \mathrm{pF}$ and a $2 \mathrm{M} \Omega$ series resistor, then distributed to each HPD via $10 \mathrm{M} \Omega$ series resistors. The cables are routed through the vessel using high voltage plugs and vacuum feed-through sockets ${ }^{9)}$. All solder connections are protected with silicone compound to reduce corona discharge.

\subsection{Readout Electronics and Data Acquisition}

The small single-photoelectron signals from each HPD (typically 3000 electrons at $12 \mathrm{kV}$ photocathode voltage) and the large number of pixel connections out of the vacuum envelope makes the readout and data acquisition system for the prototype RICH particularly challenging. For the measurements reported here a low-noise (and slow) preamplifier is used. Readout electronics appropriate for operation at LHC speed will be used in future tests. A schematic of the complete readout chain is shown in Fig. 9. Each HPD is connected to a frontend board, on which a 128-channel Viking VA2 [9] pre-amplifier/shaper/multiplexer chip is mounted. The multiplexed analogue signals from each of the seven front-end boards are transmitted via a distribution board to a VME flash-ADC system ${ }^{10)}$. The principal features of the front-end electronics and the interface to the VME control system are described in the following sections.

8) DEP model PP0100Y.

9) LEMO part No. VPP.1Y.416.CLLY-10

10) AROMA [10]: an 8-channel flash-ADC module, designed for the analogue readout of prototype silicon detectors for the CERN ATLAS experiment.

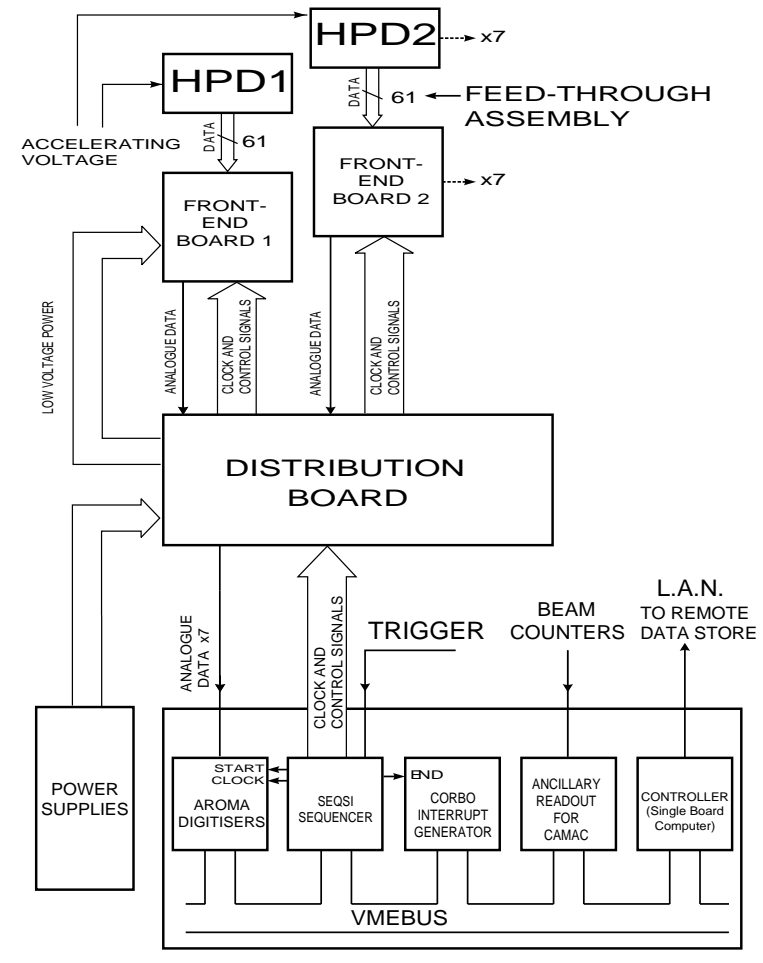

Figure 9: A schematic of the prototype RICH readout system.

\subsubsection{Electronics}

The signal pins of each HPD plug into a feedthrough assembly. This assembly of printed circuit boards is completely passive. It carries the signals from the HPD pixels, inside the RICH gas vessel, through the vessel walls to an external custom-built connector. The printed circuit boards are epoxypotted inside an aluminium tube, which seals to the machined detector plate with an O-ring.

The HPD signals are amplified and buffered close to each HPD on the front-end board, which plugs directly into the feed-through. More details of the front-end board are shown in Fig. 10. A Viking VA2 ASIC, wire-bonded to tracks on the board, is used to process the HPD-pixel signals. Each of the 61 pixel signals is input to a preamplifier and shaper on the VA2 chip. The VA2 amplification is set to approximately $55 \mathrm{mV} / \mathrm{fC}$. The output from the shaper is sampled near its peaking time of $\sim 1.2 \mu \mathrm{s}$ and stored for readout. The sample level is then read by clocking the VA2 output shift register that sequentially enables each channel level onto a differential output bus. The analogue signal outputs are then buffered with $50 \Omega$ drivers and connected via short cables to a distribution board (described below).

A low noise $\pm 2 \mathrm{~V}$ supply for the VA2 chip is generated from $\pm 5 \mathrm{~V}$ power lines, regulated using a simple transistor network. The ground of the frontend board is referenced to the chassis of the HPD via clips which attach to pins screwed into the flange of the feed-through assembly. This method provides 


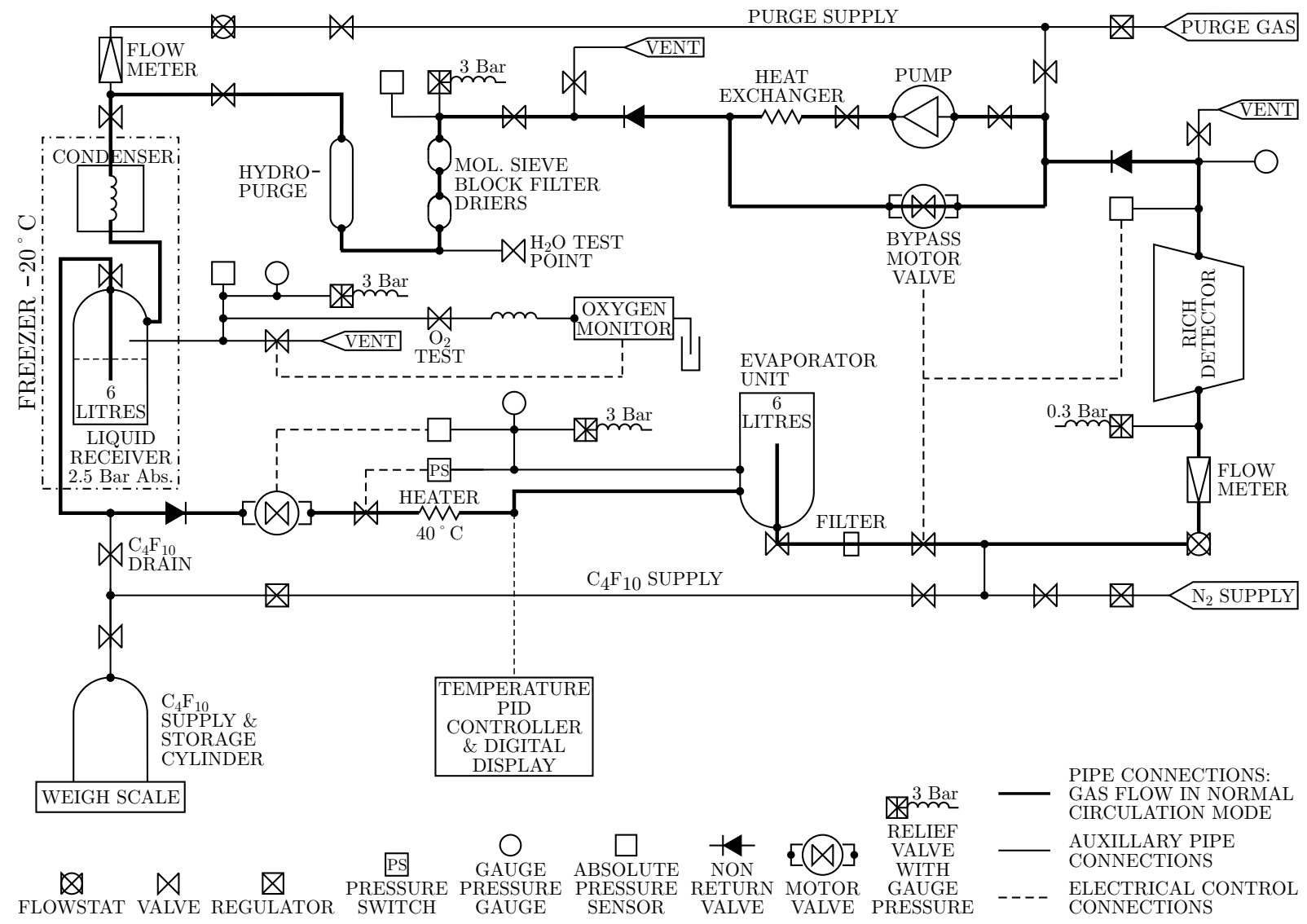

Figure 8: Schematic Gas System for Perfluorobutane $\left(\mathrm{C}_{4} \mathrm{~F}_{10}\right)$. The main circuit is indicated with the thicker line.

both a mechanically stable and a low inductance ground connection to the HPD. A photograph of an HPD, together with its feed-through and frontend boards, is shown in Fig. 11.

The distribution board interfaces the frontend boards to the data acquisition system. The board is shown schematically in Fig. 12. It receives a pattern of data from a SEQSI [11] VME sequencer module, described in section 2.7.2, then shapes and fans out these signals to the front-end boards. It receives the analogue signals from the VA2 chips, amplifies them and feeds them to the AROMA ADC module. Finally it filters the DC bias for the HPD silicon detectors and the power lines that supply the front-end boards.

The six timing and control signals from the SEQSI module are converted from ECL to TTL on the distribution board. Five of these signals are level-changed to $\pm 2 \mathrm{~V}$ using constant current devices and appropriate series resistors. The sixth TTL signal is used for the "calibrate" signal. It is reduced from TTL to $800 \mathrm{mV}$ using the diode configuration shown, then with resistor $R_{x}$ (Fig.12) set to $2.7 \mathrm{~K} \Omega$, it gives a signal equivalent to one MIP $(\sim 30,000$ electrons). All six signals are fed to each of the seven VA2 chips mounted on the front-end boards.
The analogue signal level from the VA2 is received by an NE592 amplifier (the voltage gain is 6.8 ) and buffered to drive a $120 \Omega$ shielded twistedpair cable to the VME AROMA module for digitisation. The low level signals of interest are of order one tenth of a MIP and this requires great care in the layout and decoupling within the distribution board. Similarly, the fanned-out Clock and Hold signals have their rise/fall times degraded (by incorporating $150 \Omega$ series resistors) to avoid injection of noise into the analogue signal path.

\subsubsection{Data-Acquisition System}

The readout system for the RICH prototype, shown in Fig. 9, processes data from various sources: seven HPD's, a silicon beam telescope, ADC's from beam counters, and several scalers. All are read out on every triggered event except the scalers which are read at the end of each beam spill.

In normal running conditions, the ungated beam trigger rate was $500 \mathrm{~Hz}$ during the $200 \mathrm{~ms}$ beam spill, with a $7 \mathrm{~s}$ inter-spill period. The bulk of the raw data (about $5 \mathrm{kB}$ per event) derive from the HPD's and the beam telescope.

The readout system receives a single trigger input for each beam counter coincidence. Further triggers are gated out during the readout cycle. 


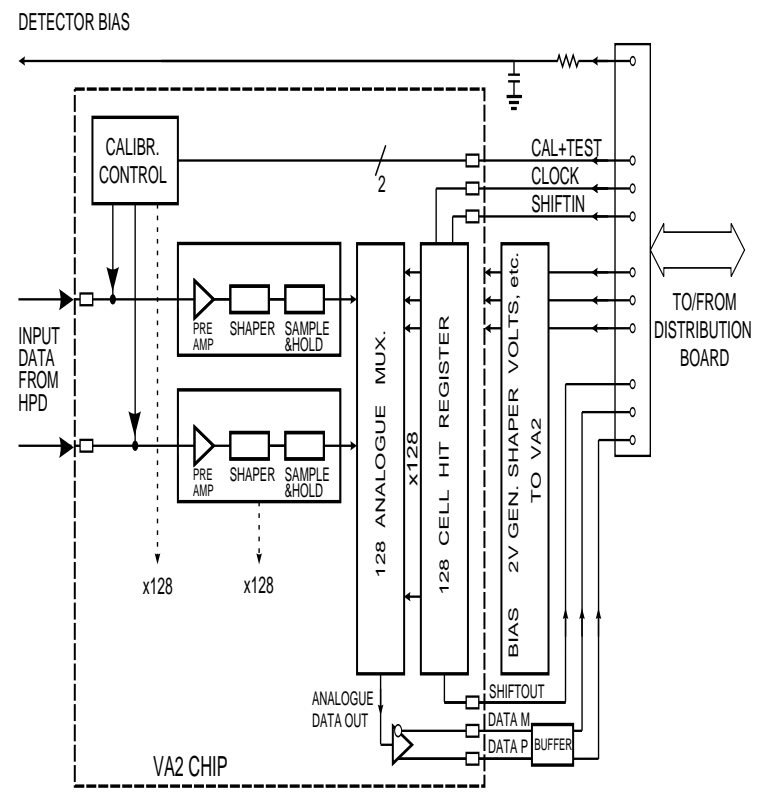

Figure 10: Front-End Board schematic.

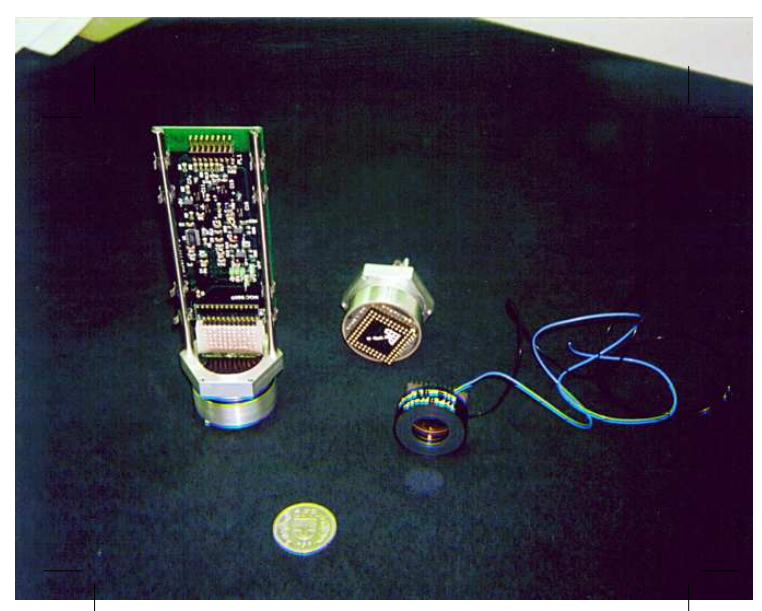

Figure 11: Photograph of an HPD, Feed-through and Front-end boards.

Control of the front-end chip must be synchronous with this trigger signal. The most important constraint is that the "hold" signal must be asserted at a constant time relative to the passage of the triggering beam particle through the detectors. The remaining time-critical constraint is that the clocking of the front-end chip and the clocking of the digitiser must be synchronised. The relative timing of these two clocks must be adjusted so that the digitiser sampling time can best match the shape of the analogue signals. The precise timing of the remaining control signals is not critical, except that the correct time ordering must be respected.

All of the above conditions are met using the SEQSI programmable front-end control module. This VME module has 20 programmable sequences each with a depth of $64 \mathrm{kbit}$ and each having an

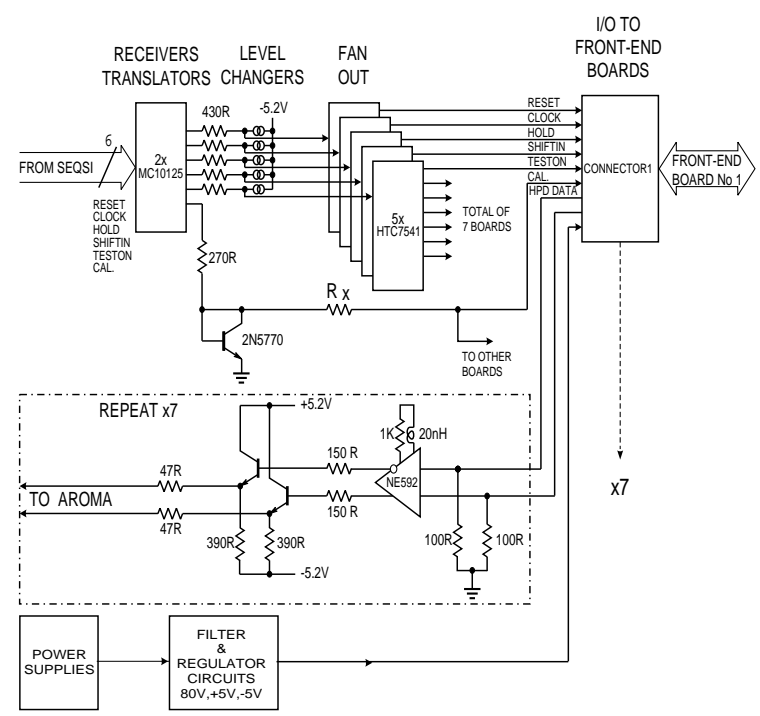

Figure 12: Schematic of the distribution board, interfacing front-end electronics to the VME.

independent differential ECL output. The module is triggered using a NIM input. Connection of the SEQSI to the VA2 chip is via a flat ribbon cable to the distribution board and thence to the frontend boards. The clocking signals for the digitisers are also generated directly by the SEQSI and connected to the digitiser modules using level adapters where necessary.

The front-end chips output a multiplexed serial stream of analogue information during the readout cycle. The HPD's generate seven independent streams each containing 128 analogue levels. These are digitised using the 8-channel AROMA VME digitiser.
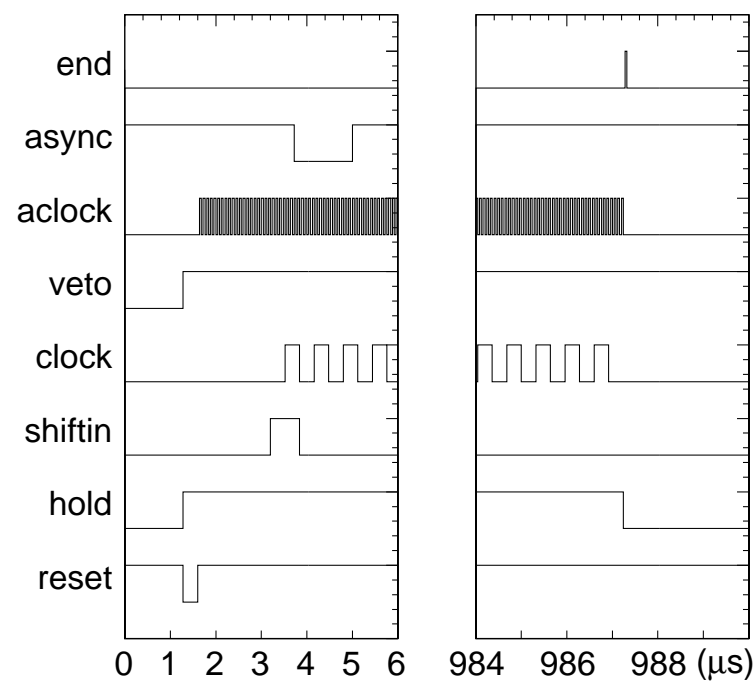

Figure 13: Timing sequence for the readout.

The sequence of signals generated by the SEQSI during a normal readout cycle is shown in 
Fig. 13. When no triggers are generated the SEQSI executes an "idle loop" in which none of the outputs change state. Shortly after a trigger signal is received, the SEQSI executes the readout sequence. The readout sequence starts with "reset" which resets the digital logic of the front-end chips. At the same time "hold" is asserted. This holds the output from the front-end shapers in preparation for multiplexing onto the output stream. Following the hold signal, the "shiftin" signal initialises the operation of the front-end multiplexers. The multiplexers are then clocked with "clock" and the AROMA digitiser is clocked with "aclock" which is divided internally in the AROMA. The dividing of the AROMA clock allows the phase of the digitiser sampling to be adjusted relative to "clock", in steps of 50ns, by programming of the SEQSI sequence. The "async" pulse is required by the AROMA to initialise the digitisation cycle. The SEQSI also provides a "veto" to inhibit further triggers during the readout cycle.

Finally an "end" signal is generated and is routed to a $\mathrm{CORBO}^{11)}$ module which generates a VME interrupt. An interrupt handler executed in a VME single board computer ${ }^{12)}$ performs the concatenation of the raw data streams from the HPD's, the beam telescope, a beam Cherenkov detector and the scalers, and re-enables the SEQSI into its idle loop in preparation for the next trigger.

The data relating to one event are assembled using standard $\mathrm{C} / \mathrm{C}++$. Binary files, with zero suppression applied to the beam telescope data, are stored in RAM on the VME processor and are then copied via Ethernet to a hard disk once the memory buffer is full.

\subsection{Light-Emitting Diode diagnostic system}

The complete readout and data acquisition chain was tested using a weak source of light from a pulsed light-emitting diode (LED). It was placed at the beam entrance window and, following reflection from the mirror, illuminated the photocathodes of all HPD's. The data acquisition system was synchronised with the LED pulser. The resulting pulse-height spectrum for one HPD pixel is shown in Fig. 14. The noise peak (pedestal), the single, double and triple photo-electron peaks are clearly visible. Gaussians were fitted to the pedestal, single and multiple photo-electron peaks in order to measure the signal pulse height and the noise. Spectra from all other pixels have similar Gaussian parameters indicating a uniform response over the photocathode of the HPD and among the different HPD's. The signal/noise ratio is typically $\sim 5.7$, corresponding to a noise charge of approximately 500 electrons. Most of this noise is associated with

\footnotetext{
11) CES RCB8047. Address: CES, 1213 Petit-Lancy,CH.

12) CES RIO, model No.8061.
}

the input capacitance due to the feed-through and printed-circuit boards connecting the pixels to the VA2 chip.

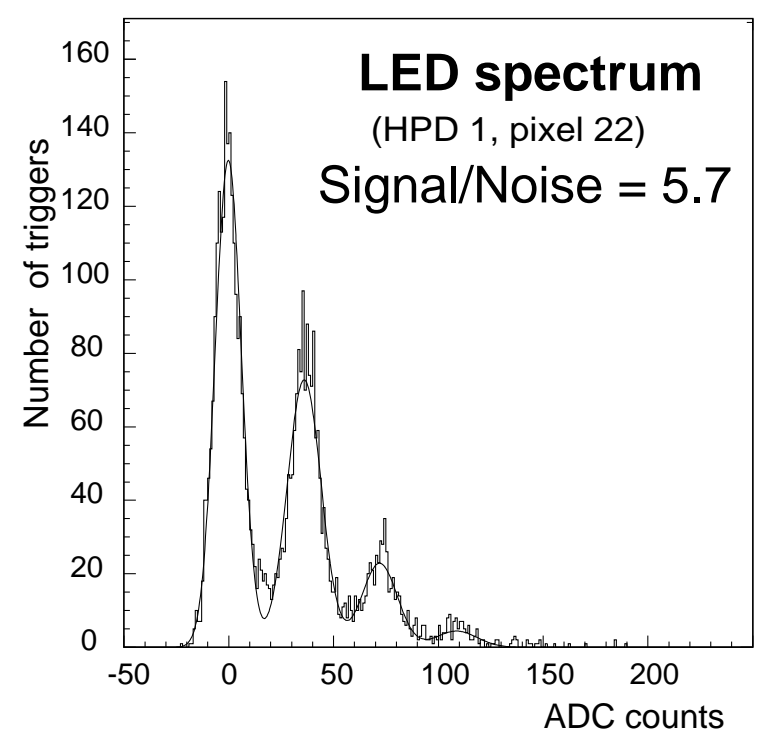

Figure 14: The pulse height spectrum obtained from a single HPD pixel, using an LED source.

\section{$3 \quad$ Measurements in a test beam}

The RICH prototype was set up in the T9 test beam at the CERN PS. Data were collected during Spring/Summer 1997. The first results, following from analysis of the data, are described in this section.

\subsection{The test beam setup}

The T9 test facility is a secondary beam, derived from extracted CERN PS protons, and provides charged particles of either polarity. The momentum can be tuned in the range $2-15.5 \mathrm{GeV} / c$. The particle type $(e, \pi, \mathrm{K}, \mathrm{p})$ is identified by measuring the signal pulse height from a $\mathrm{CO}_{2}$ threshold Cherenkov counter, installed $30 \mathrm{~m}$ upstream of the prototype.

The prototype vessel is aligned on the beam axis. Charged particles which provide the trigger are selected using scintillation counters, two upstream and two downstream of the vessel. The smallest scintillator $\left(4 \times 4 \mathrm{~mm}^{2}\right)$ defines the accepted beam size. During one typical extraction spill of $200 \mathrm{~ms}$ duration, $5 \times 10^{3}$ beam particles enter the detector volume, resulting in $\sim 30$ triggers accepted by the data acquisition. The beam trajectory is measured using a telescope consisting of three planes of silicon-pad detectors. The pad dimensions are $1.3 \times 1.3 \mathrm{~mm}^{2}$, and the planes are placed upstream of the vessel and separated by approximately one metre. 


\subsection{Observation of Cherenkov rings}

Using RICH configuration-1, data were taken with a $10 \mathrm{GeV} / c$ negatively charged beam. The vessel initially contained air, which was used as the gaseous radiator, and $18 \mathrm{~mm}$ thickness of aerogel (Sample-1) was placed in the beam, at the entrance to the RICH detector.

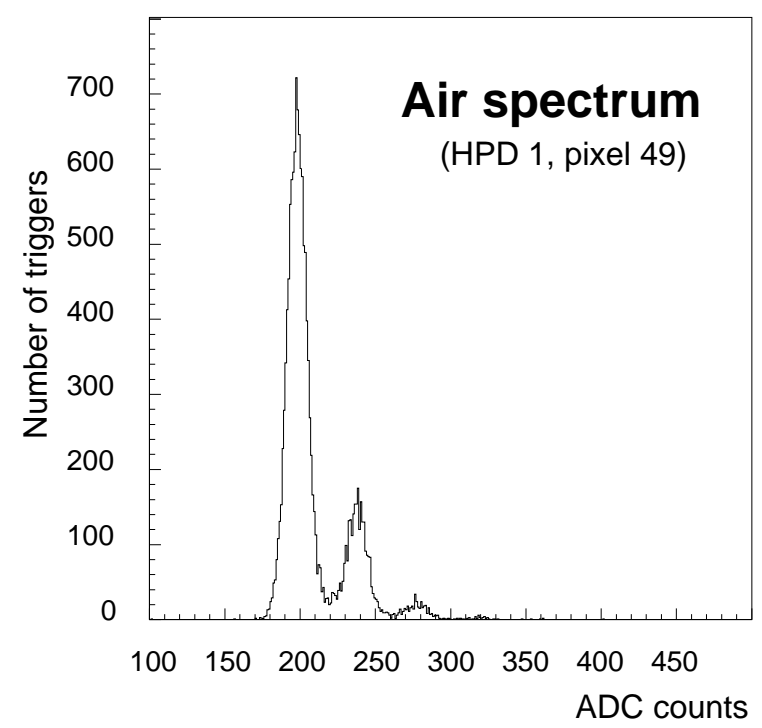

Figure 15: ADC spectrum for a pixel on the air ring.

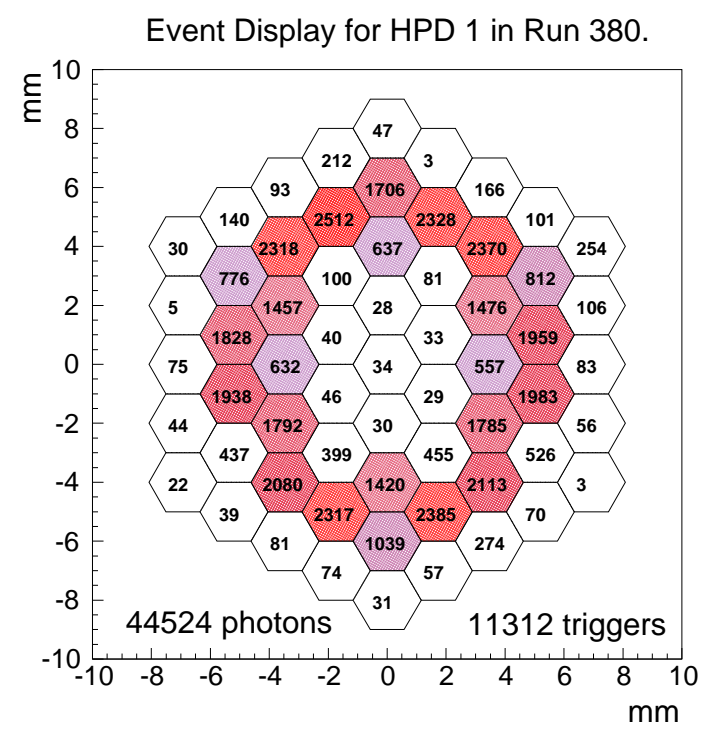

Figure 16: Cherenkov ring from the air radiator: an event display for the central HPD.

Fig. 15 shows an accumulated ADC spectrum for an HPD pixel which lies on the Cherenkov ring from the air radiator. For every pixel channel the r.m.s width of the pedestal distribution, the mean of the single-photoelectron peak and its separation above the pedestal mean were determined by fitting multiple Gaussians to the spectra obtained from dedicated pedestal runs and from runs with a pulsed LED source. For this first analysis a photoelectron hit is defined to be an HPD pixel with a signal pulse height $4 \sigma$ above the pedestal mean, where $\sigma$ is the r.m.s width of the pedestal peak.

The pixels that lie on the air ring have a typical mean number of photoelectrons of $0.2-0.3$ per pixel per event. The spectra of "off-ring" pixels are indistinguishable from typical pedestal distributions. For pixels on the aerogel ring the mean number of photoelectrons per pixel per event is $\simeq 0.02$, about a factor 10 smaller than is observed for the air ring.

A display of the central HPD is shown in Fig. 16. Using the criteria described above, the numbers of photoelectrons are counted and displayed for each pixel. The values are indicated by the shading of the pixels. A clear ring is visible; there is typically a factor of more than 20 difference between the number of pixels hit on the ring and those off the ring.

Replacing the air radiator with $\mathrm{C}_{4} \mathrm{~F}_{10}$, the larger Cherenkov angle results in a ring which is no longer contained within the central HPD. Fig. 17 shows an arc of the ring on the central HPD, whose radius is compatible with that expected from the refractive index of $\mathrm{C}_{4} \mathrm{~F}_{10}$ (1.0014). The outer HPD's clearly exhibit a ring which originates from the aerogel radiator.

\section{Event Display of Run 487 (6225 triggers)}

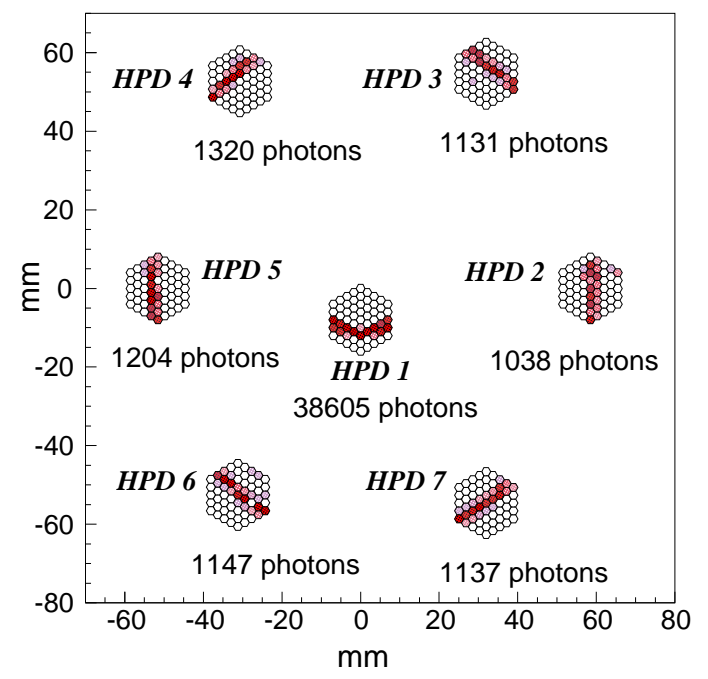

Figure 17: An event display from aerogel and $\mathrm{C}_{4} \mathrm{~F}_{10}$ radiators in RICH configuration-1.

The number of photoelectrons per triggered event was measured for each radiator, and compared with expectations using a simulation of the prototype. For this photoelectron counting analysis, a threshold of $3 \sigma$ was set for individual pixels. This requirement resulted in a $15 \pm 5 \%$ loss from the single photoelectron peak, averaged over all the chan- 
nels. Multiple photoelectron hits were taken into account by applying a correction calculated from the higher multiplicity peaks visible in the ADC spectra (Fig. 15). The mean number of photoelectrons per event was counted for pixels on the air ring, the aerogel ring and the $\mathrm{C}_{4} \mathrm{~F}_{10}$ ring. These numbers are shown in Table 1.

The background to the photoelectron signal was estimated using pixels situated far from the Cherenkov ring. The procedure was cross-checked by performing the same analysis on the dedicated pedestal runs, thus isolating that component resulting from electronic noise, and by using simulation to estimate the contribution from photons Rayleighscattered by the aerogel. The total background from these two sources was compatible with that from the direct measurement using the off-ring pixels. For the air and $\mathrm{C}_{4} \mathrm{~F}_{10}$ radiators the background correction corresponds to $2 \%$ of the signal, whereas for the aerogel it is $25 \%$.

The measured photoelectron yields were corrected for the effects described above and also for the partial geometrical coverage of the aerogel and $\mathrm{C}_{4} \mathrm{~F}_{10}$ rings, approximately $20 \%$ and $25 \%$ respectively. These geometrical factors were calculated from simulation with $\sim 5 \%$ uncertainty. Table 1 lists the number of photoelectrons per event after all corrections.

The expected photoelectron yield was calculated from simulations which used our measurements, as a function of wavelength, of the aerogel clarity, the mylar window transmission and the mirror reflectivity. The quantum efficiencies of the HPD's were measured by the manufacturer (the $\int Q d E$ is approximately $1.3 \mathrm{eV}$ ). The overall precision in the expected yield, including uncertainties in these characteristics, is estimated to be $15 \%$.

The comparison between observation and expectation is given in Table 1 . The numbers from this preliminary analysis are compatible within $30 \%$, indicating a basic understanding of the Cherenkov light production and detection in the prototype. The photon yield from the air and $\mathrm{C}_{4} \mathrm{~F}_{10}$ radiators correspond to a figure of merit $N_{0} \simeq 250 \mathrm{~cm}^{-1}$, where $N_{0}$ is defined in relation to the number of detected photoelectrons

$$
N_{\text {photoelectrons }}=N_{0} \sin ^{2} \theta_{\mathrm{C}} L
$$

for a radiator of length $L$. The photon yields (with borosilicate glass filter) from the aerogel samples indicate a corresponding value for $N_{0} \simeq 50 \mathrm{~cm}^{-1}$.

The full-scale RICH-1 prototype was studied using configuration-2. Due to the longer focal length mirror, the ring from $\mathrm{C}_{4} \mathrm{~F}_{10}$ spans the outer six HPD's. The event display shown in Fig. 18 is obtained from data taken with a negatively-charged beam of momentum $15.5 \mathrm{GeV} / c$. The $\mathrm{K}: \pi$ ratio of the beam particles which trigger the readout was enhanced to $1: 2$ using the information from the

\begin{tabular}{|l|l||l|c|c||c|}
\hline Radiator & $\begin{array}{l}\text { Filter } \\
\text { used }\end{array}$ & $\begin{array}{l}\text { Raw } \\
\text { hits }\end{array}$ & $\begin{array}{c}\text { Bkg. } \\
\text { corr. }\end{array}$ & $\begin{array}{c}\text { Eff. } \\
\text { corr. }\end{array}$ & Ratio \\
\hline Air & & 4.92 & 4.56 & 4.80 & 0.99 \\
\hline $\mathrm{C}_{4} \mathrm{~F}_{10}$ & & 7.85 & 7.49 & 33.55 & 1.07 \\
\hline Aerogel 1 & & 1.79 & 1.31 & 10.71 & 0.72 \\
Aerogel 1 & glass & 1.14 & 0.75 & 6.14 & 0.92 \\
Aerogel 1 & mylar & 1.34 & 0.81 & 3.54 & 0.75 \\
\hline Aerogel 2 & glass & 0.85 & 0.52 & 4.62 & 1.10 \\
\hline
\end{tabular}

Table 1: Observed number of photoelectrons per event for air, aerogel and $\mathrm{C}_{4} \mathrm{~F}_{10}$ radiators. The columns give the numbers of raw hits, the numbers after correction for background and for geometrical efficiency, and in the final column, the comparison with the expected yield.

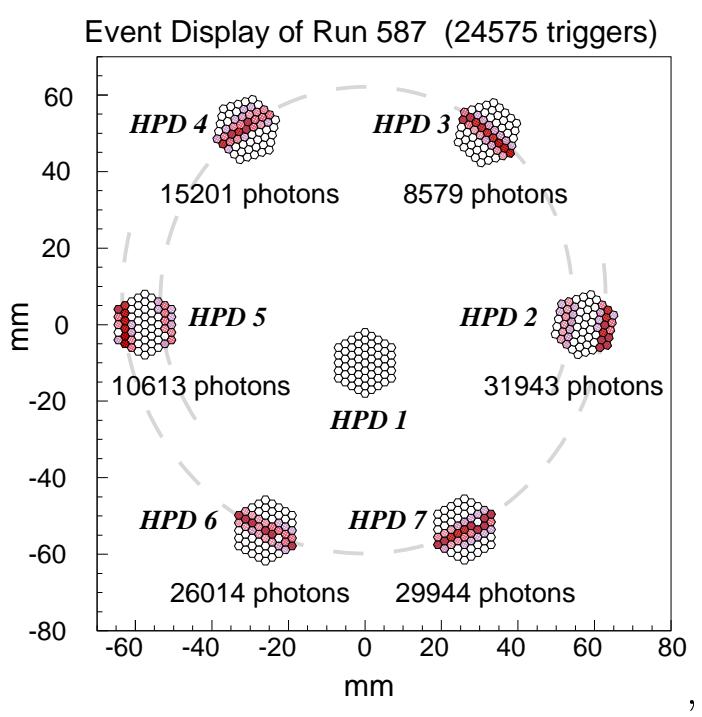

Figure 18: An event display showing $\pi / \mathrm{K}$ separation, using $\mathrm{C}_{4} \mathrm{~F}_{10}$ radiator in $\mathrm{RICH}$ configuration- 2 .

beam threshold Cherenkov counter. Fig. 18 shows segments of two Cherenkov rings; an inner ring, from the incident kaons, and an outer ring from the pions. Note that the number of hits observed in HPD 3 is lower than in HPD 4; similarly HPD 5 has fewer hits than HPD 2. The reason for the asymmetry is because HPD's 3 and 5 have mylar windows in front of their photocathodes which absorb the UV photons.

\section{Summary}

Cherenkov ring images from gas and aerogel radiators have been clearly observed using HPD's as photon detectors. The HPD's show very good single-photon pulse height resolution and pixel-topixel uniformity. The HPD's and their read-out proved relatively trouble-free over three months of operation. During this period 5 million triggers were recorded with more than $96 \%$ of pixel channels fully operational.

The measured photon yields from the gas ra- 
diators (air and $\mathrm{C}_{4} \mathrm{~F}_{10}$ ) are in good agreement with expectations based on simulation. The photon yield from the KEK aerogel sample is approximately $20 \%$ less than that expected from its measured clarity and further work is necessary to understand this.

Analysis of the data is underway to reconstruct, for each recorded hit, the Cherenkov emission angle, both from gas and aerogel radiators. The precision achieved, combined with the measurements of the detected photon yield, will allow an evaluation of the particle identification performance of the LHC-B RICH detector.

\section{Acknowledgements}

The work reported here would not have been possible without the technical support provided by colleagues from our home institutes. We thank, in particular, H.Baughan, D.Campbell, I.Clark, P.Evans, S.Greenwood, S.Johnson, V.Kasey, R.Knott, C.Raine, and M.Rankin for their contributions to the construction of the prototype $\mathrm{RICH}$ and its associated gas system and read-out electronics. Colleagues from the LHC-B Collaboration have provided valuable advice and assistance during the beam tests at CERN, especially, R.Forty, G.McEwen, O.Ullaland and T.Ypsilantis. The silicon beam telescope was provided for us as a working system by E.Chesi and J.Seguinot. E.Nappi provided some of the aerogel samples and advised on its handling. Finally, we gratefully acknowledge the CERN PS division for their provision of the test beam and the UK Particle Physics and Astronomy Research Council for their financial support.

\section{References}

[1] LHC-B Letter of Intent, "A Dedicated LHC Collider Beauty Experiment for Precision Measurements of CP Violation", CERN/LHCC 95-5.

[2] R.Forty, Nucl. Instr. and Meth. A 384 (1996) 167.

[3] T.Ypsilantis and J.Seguinot, Nucl Instr. and Meth. A 368 (1995) 229.

[4] R. De Salvo et al., Nucl. Instr. and Meth. A 315 (1992) 375.

[5] E. Chesi et al., "Performance of a 256-pad Hybrid Photon Detector for ring imaging", Nucl. Instr. and Meth. A 387 (1997) 122.

[6] D.E. Fields et al., Nucl. Instr. and Meth. A 349 (1994) 431.

[7] R. deLeo et al., "Electronic Detection of focussed Cherenkov rings from Aerogel", CERN-LAA/97-02. (Submitted to Nucl. Instr. Meth. A)

[8] W. Adam et. al., Nucl. Instr. and Meth. A338 (1994) 284.

[9] O.Toker et al., Nucl. Instr. and Meth. A340 (1994) 572 .
[10] A.Baird at al., Rutherford Appleton Laboratory: AROMA manual (unpublished).

[11] M.Morrisey, Rutherford Appleton Laboratory: SEQSI manual (unpublished). 